\title{
Slip Deformation Analysis Based on Full Constraints Model for $\alpha$-Titanium Alloy at Low Temperature*
}

\author{
Motoaki Morita $^{1}$ and Osamu Umezawa ${ }^{2}$ \\ ${ }^{1}$ Graduate School of Mechanical Engineering and Materials Science, Yokohama National University, Yokohama 240-8501, Japan \\ ${ }^{2}$ Department of Materials Science and Engineering, Yokohama National University, Yokohama 240-8501, Japan
}

\begin{abstract}
The effects of restricted slip conditions on both the Taylor factor and plastic work rate under the condition of tensile yielding have been analyzed in $\alpha$-titanium alloys at low temperatures, using the full constraints model. The role of secondary slip systems, i.e., the $\langle a\rangle$ basal slip and $\langle c+a\rangle$ pyramidal slip, was clarified, when the $\langle a\rangle$ prismatic slip was dominant. Although no influence of secondary slip conditions on the Taylor factor was detected, the plastic work rate was sensitive to the operating secondary slip systems. When the basal system was chosen as the secondary slip system, the plastic work rate increased in all tensile axes, especially around $\langle 0001\rangle$. In addition, no basal slip operation decreased the plastic strain energy. The plastic work rate was the highest along the $\langle 0001\rangle$ tensile axis, and the operation of the $\langle c+a\rangle$ pyramidal slip was necessary to achieve plastic deformation along $c$ axis. High elastic strain energy, therefore, must accumulate to a high level around $\langle 0001\rangle$, because the pyramidal slip is hardly active owing to its very high critical resolved shear stress. [doi:10.2320/matertrans.L-M2011815]
\end{abstract}

(Received February 14, 2011; Accepted April 30, 2011; Published July 25, 2011)

Keywords: Taylor factor, critical resolved shear stress, hexagonal close packed structure, fatigue, primary slip

\section{Introduction}

Subsurface crack generation in the high-cycle fatigue of $\alpha$-titanium alloys is dominant at low stress regimes and temperatures. ${ }^{1)}$ The crack initiation sites appear at crystallographic facets such as the (0001) transgranular cracking. ${ }^{2-5)}$ The dislocation movement of the alloys is nearly planar and $\{01 \overline{1} 0\}\langle 11 \overline{2} 0\rangle$ arrays pile up near grain boundaries. ${ }^{2)}$ The local stress concentration near a grain boundary due to heterogeneous slip may lower the high-cycle fatigue strength and cause the subsurface crack initiation. Therefore, it is necessary to consider crystal plasticity in the cyclic deformation and fatigue fracture mechanism. Here, the fatigue process is divided into four stages: (1) development of a saturated dislocation structure by cyclical microplastic strain accumulation, (2) generation of localized slip and/or microcracking to relax the stress concentration at the vicinity of a boundary, (3) microcrack growth and transition to main crack, and (4) crack propagation. ${ }^{3)}$ In stages 3 and 4, linear mechanics can be applied to evaluate the critical size of the subsurface crack (facet) for propagation ${ }^{6)}$ and the crack propagation life. ${ }^{7}$ However, stages 1 and 2, which are based on estimated models, do not exhibit direct evidence of cracking and macroscopic support. Thus, the evaluation based on crystal plasticity complements the construction of a sophisticated model of subsurface fatigue crack initiation.

In stage 1 , the $\{01 \overline{1} 0\}\langle 11 \overline{2} 0\rangle$ dominant slip operates in some grains; this slip depends on the crystal orientation. Moreover, both plastic and elastic deformations coexist in polycrystalline specimens. In stage 2 , regardless of whether the origin of transgranular cracking is slip-off (slip localization) or microcracking on a crystal plane, the plastic deformation on the facet plane hardly yields and the elastic field normal to the facet plane has to accumulate. The activity

*This Paper was Originally Published in Japanese in J. JILM 60 (2010) 61-67. In Regard to the Paper, the Erratum was Published on Page 296, Vol. 61 of J. JILM. of slips, needed for any micro-yielding, can be evaluated on the basis of the full constraints model using Taylor analysis. ${ }^{8,9)}$ Subsequently, the deformation behavior of the secondary slips in a locally yielded grain describes the elastic (stress) field in stages 1 and 2. The full constraints model is based on the assumption of homogeneous deformation in polycrystalline specimens and has been mainly applied to understand the homogeneous and large-scale deformation in face-centered cubic (f.c.c.) metals. ${ }^{8,9)}$ Occasionally, the full constraints model has been applied to slip analysis in hexagonal closed packed (h.c.p.) metals, because twinning has a great influence on their homogeneous and large-scale deformation. ${ }^{10)}$ In the present study, we adopted the full constraints model in order to evaluate the critical resolved shear stresses (CRSSs) in the primary slip system of $\alpha$ titanium at low temperatures. In addition, we calculated the external (internal) plastic work rate and Taylor factor to discuss the effect of the primary slip system on slip behavior and that of the crystal orientation on the stress field.

\section{Procedure}

\subsection{Application of full constraints model}

A grain in the tensile stress field that develops by cyclic deformation is constrained by its neighbors in a polycrystalline specimen. The full constraints model, in which all the grains in the field exhibit the same strain, was adopted to sustain the strain compatibility between neighboring grains. That is, to satisfy the full constraints, multiple slips were introduced in a grain. In the tension mode, the compatibility in a polycrystalline specimen can be achieved by operating five independent slip systems. ${ }^{8,9)}$ When an uniaxial tensile strain is parallel to the $z$-axis in the specimen's coordinate system, XYZ, grain deformation takes place under axial symmetry at a fixed volume $\left(\mathrm{d} \varepsilon_{x x}+\mathrm{d} \varepsilon_{y y}+\mathrm{d} \varepsilon_{z z}=0\right):{ }^{11)}$

$$
\begin{aligned}
& \mathrm{d} \varepsilon_{x x}=\mathrm{d} \varepsilon_{y y}=-\frac{1}{2} \mathrm{~d} \varepsilon_{z z} \\
& \mathrm{~d} \gamma_{x y}=\mathrm{d} \gamma_{y z}=\mathrm{d} \gamma_{z x}=0
\end{aligned}
$$


where $\mathrm{d} \varepsilon_{x x}, \mathrm{~d} \varepsilon_{y y}$ and $\mathrm{d} \varepsilon_{z z}$ are the plastic strain rate, and $\mathrm{d} \gamma_{x y}$, $\mathrm{d} \gamma_{y z}$ and $\mathrm{d} \gamma_{z x}$ are the plastic shear strain rate in a grain. The internal plastic work rate $\mathrm{d} w$ is the increment of work per volume and it is the sum of the work of five independent slip systems in a grain:

$$
\mathrm{d} w=\sum_{i} \tau \mathrm{d} \gamma_{i}=\tau \sum_{i}\left|\mathrm{~d} \gamma_{i}\right| \quad(i=1,2, \cdots, 5)
$$

where $\tau_{i}$ is the CRSS and $\mathrm{d} \gamma_{i}$ is the slip rate in the $i$-th slip system. There are a number of combinations of operating slip systems that satisfy the external work constraint, but only one combination is to be chosen. Each time ideal plastic deformation take places, $\mathrm{d} w$ equals the external plastic work rate as in eq. (4):

$$
\mathrm{d} w=\tau \mathrm{d} \gamma=\sigma_{z z} \mathrm{~d} \varepsilon_{z z}
$$

The Taylor factor $M$ is calculated using eq. (4) as

$$
\frac{\sigma_{z z}}{\tau}=\frac{\mathrm{d} \gamma}{\mathrm{d} \varepsilon_{z z}}=M
$$

$M$ represents the total of the slip rates in five independent slip systems for the strained grain, and depends on the relationship between the tensile axis (z-axis) and grain orientation.

\subsection{Evolution of full constraints model applied to low- temperature slip deformation in $\alpha$-titanium}

\subsubsection{Coordinate transformation in hexagonal lattice}

The strain rate tensor $\mathrm{d} \varepsilon_{i j}$ in the specimen space is equal to the strain rate tensor $\sum_{k} E_{i j}^{k}$, which is the sum of the strain rate tensors in five independent slip systems:

$$
\mathrm{d} \varepsilon_{i j}=\left(\begin{array}{lll}
\mathrm{d} \varepsilon_{x x} & \mathrm{~d} \gamma_{x y} & \mathrm{~d} \gamma_{x z} \\
\mathrm{~d} \gamma_{y x} & \mathrm{~d} \varepsilon_{y y} & \mathrm{~d} \gamma_{y z} \\
\mathrm{~d} \gamma_{z x} & \mathrm{~d} \gamma_{z y} & \mathrm{~d} \varepsilon_{z z}
\end{array}\right)=\sum_{k} E_{i j}^{k} \quad \begin{aligned}
& (i, j=1,2,3) \\
& (k=1,2, \cdots, 5)
\end{aligned}
$$

In the specimen coordinate system, the $E_{i j}^{k}$ are described by the slip direction vector $\boldsymbol{b}$ and the normal vector direction to the slip plane $\boldsymbol{n}$, where the coordinate transformation from the Miller-Bravais index in the crystal to the orthogonal coordinate system in the specimen was done under the coordinates shown in Fig. 1. First, $\boldsymbol{b}^{\mathrm{mbi}}[i j k h]$ given by the Miller-Bravais index (axis: $a^{1}, a^{2}, a^{3}, c$ ) was transformed into $\boldsymbol{b}^{\text {norh }}[(i-k)(i-j)(c / a) \times h]$ in the nonorthogonal coordinate system (axis: $A^{1}, A^{2}, C, \gamma=120^{\circ}$ ), and then $b^{\text {orh }}$ in the orthogonal one (axis: $\left.x^{\prime}, y^{\prime \prime}, z^{\prime}\right)$ as

$$
\boldsymbol{b}^{\text {orh }}=\boldsymbol{Q} \cdot \boldsymbol{b}^{\text {norh }}
$$

$\boldsymbol{Q}$ is the coordinate transformation tensor which represents the relationship between the unit vector of axes in the nonorthogonal coordinate system $\left(A^{1}, A^{2}, C\right)$ and that in the orthogonal one $\left(x^{\prime}, y^{\prime \prime}, z^{\prime}\right)$. Second, the normal vector to the slip plane $\boldsymbol{n}^{\text {orh }}$ was estimated from the exterior product of the two slip directions $\boldsymbol{b}_{1}^{\text {orh }}$ and $\boldsymbol{b}_{2}^{\text {orh }}$ on a slip plane as

$$
\boldsymbol{n}^{\text {orh }}=\boldsymbol{b}_{1}^{\text {orh }} \times \boldsymbol{b}_{2}^{\text {orh }}
$$

Subsequently, the $\boldsymbol{n}^{\text {orh }}$ and $\boldsymbol{b}^{\text {orh }}$, represented in the orthogonal coordinate system in the crystal, were transformed to the $\boldsymbol{n}$ and $\boldsymbol{b}$ in the orthogonal coordinate system in the specimen as eq. (9):

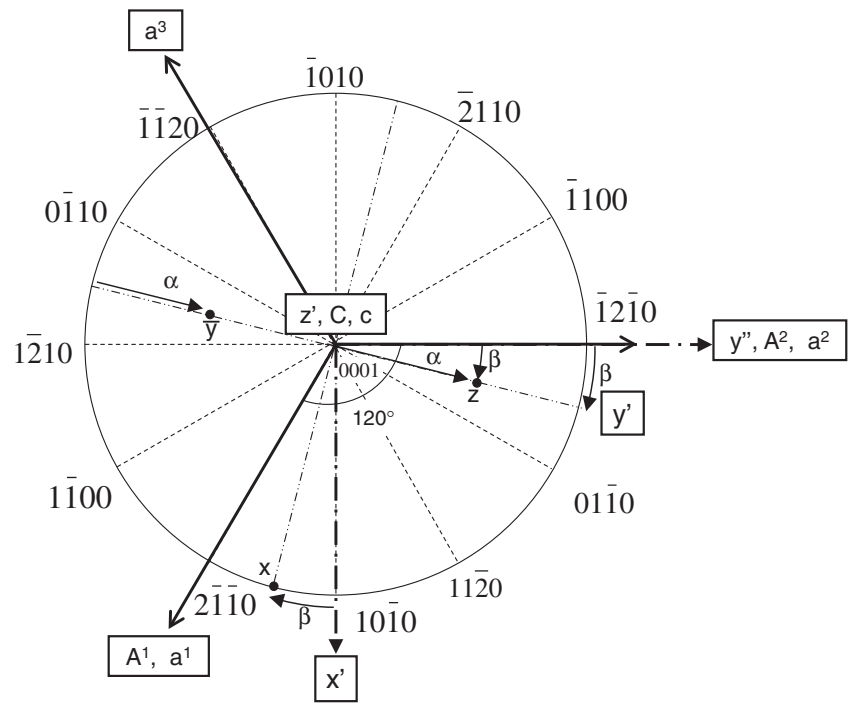

Fig. 1 Definition of $\alpha$ and $\beta$ on the standard stereographic projection of (0001) plane with Miller-Bravais index $\left(a^{1}, a^{2}, a^{3}, c\right)$, orthogonal coordinates system $\left(x^{\prime}, y^{\prime \prime}, z^{\prime}\right)$, and nonorthogonal coordinates system $\left(A^{1}, A^{2}, C\right)$ in crystal and orthogonal coordinates system $(x, y, z)$ in specimen.

$$
\begin{aligned}
\left(n_{i} \text { or } b_{i}\right)= & \left(\begin{array}{ccc}
\cos \beta & -\sin \beta & 0 \\
\cos \alpha \sin \beta & \cos \alpha \cos \beta & -\sin \alpha \\
\sin \alpha \sin \beta & \sin \alpha \cos \beta & \cos \alpha
\end{array}\right) \\
& \times\left(n_{i}^{\text {orh }} \text { or } b_{i}^{\text {orh }}\right)
\end{aligned}
$$

where angular parameters $\alpha$ and $\beta$ represent the transformation from the orthogonal coordinates in the crystal to those in the specimen, $x y z$, (Fig. 1). The rotation angle $\alpha$ $\left(0^{\circ}<\alpha<90^{\circ}\right)$ along the $x$-axis in the orthogonal coordinates, $x y^{\prime} z^{\prime}$, gives the transform into the orthogonal coordinates, xyz. The angle $\beta\left(0^{\circ}<\beta<90^{\circ}\right)$ along $z^{\prime}$-axis in the orthogonal coordinates, $x^{\prime} y^{\prime \prime} z^{\prime}$, gives the transform into the $x y^{\prime} z^{\prime}$.

\subsubsection{Influence of critical resolved shear stress}

Only the principal slip systems, i.e., $\{01 \overline{1} 0\}\langle 11 \overline{2} 0\rangle$, $\{0001\}\langle 11 \overline{2} 0\rangle$, and $\{10 \overline{1} 1\}\langle 11 \overline{2} 3\rangle$ were taken into account for the deformation mode in $\alpha$-titanium alloy. ${ }^{13-16)}$ In addition, no deformation twinning and $\varepsilon$-martensite were considered. Because the CRSSs in the slip systems are different from each other, especially at low temperatures, ${ }^{10,12-14,16)}$ eqs. (3) and (5) are modified to permit the multiple slips as given by eqs. (10) and (11) under the full constraints model:

$$
\begin{aligned}
& \mathrm{d} w=\sum_{i} \tau_{i}\left|\mathrm{~d} \gamma_{i}\right| \quad(i=1,2, \cdots, 5) \\
& \frac{\sum_{i}\left|\mathrm{~d} \gamma_{i}\right|}{\mathrm{d} \varepsilon_{z z}}=M \quad(i=1,2, \cdots, 5)
\end{aligned}
$$

where $M$ is the newly defined sum of the slip rates in the operating slip systems within a grain per unit strain, because it cannot be directly found by the yield stress. In a randomly oriented polycrystal under uniaxial tension parallel to the $z$-axis, the deformation is denoted as 
Table 1 Ratios in critical resolved shear stresses of major slip systems. (reference: $\{01 \overline{1} 0\}\langle 11 \overline{2} 0\rangle$ at $300 \mathrm{~K}$ )

\begin{tabular}{cccc}
\hline \multirow{2}{*}{ Type } & \multicolumn{3}{c}{ Ratio of CRSS } \\
\cline { 2 - 4 } & $\{0001\}\langle 11 \overline{2} 0\rangle$ & $\{01 \overline{1} 0\}\langle 11 \overline{2} 0\rangle$ & $\{10 \overline{1} 1\}\langle 11 \overline{2} 3\rangle$ \\
\hline 1 & 1 & 1 & 1 \\
\hline 2 & 10 & 1 & 10 \\
\hline 3 & 10 & 1 & 15 \\
\hline 4 & 15 & 1 & 22 \\
\hline 5 & 4 & 1 & 30 \\
\hline 6 & 13.5 & 3 & \\
\hline
\end{tabular}

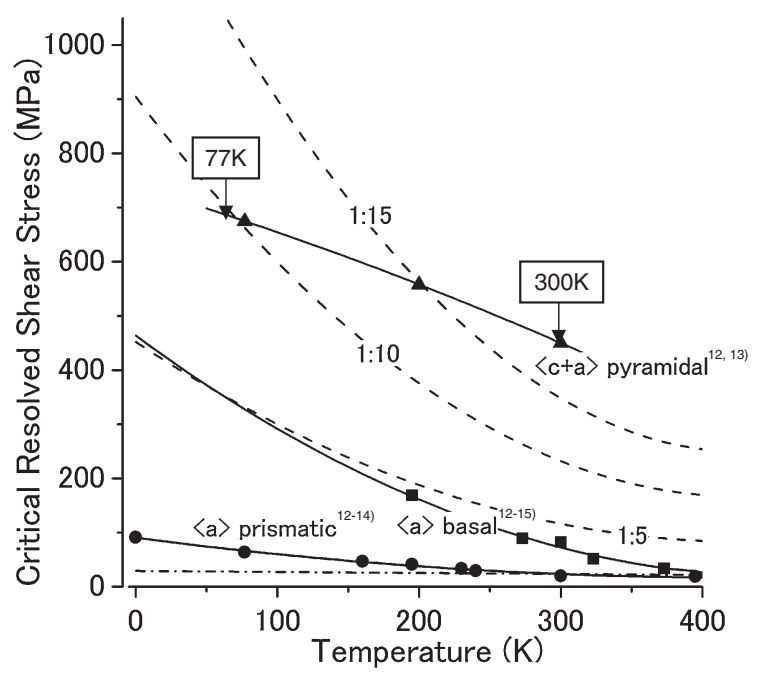

Fig. 2 Relationship between critical resolved shear stress (CRSS) and temperature for $\langle a\rangle$ prismatic, $\langle a\rangle$ basal, and $\langle c+a\rangle$ pyramidal slips in $\alpha$ Ti. Dashed curved lines indicate scales with 5, 10, and 15 times as large as the CRSS of $\langle a\rangle$ prismatic slip. The dashed-dotted line indicates the athermal component of $\langle a\rangle$ basal slip in pure Ti. ${ }^{15)}$

$$
\sum_{i} \tau_{i}\left|\mathrm{~d} \gamma_{i}\right|=\sigma_{z z} \mathrm{~d} \varepsilon_{z z} \quad(i=1,2, \cdots, 5)
$$

To evaluate the effect of the CRSSs in the principal slip systems on $M$ and $\mathrm{d} w$, six types of models were examined (Table 1). In Type 1 , the CRSSs of the three active slip systems are the same. In Types $2-6,\{01 \overline{1} 0\}\langle 11 \overline{2} 0\rangle$ is fixed as the primary slip system. Types $2-4$ simply represent the act of choosing the secondary slip system from $\{0001\}\langle 11 \overline{2} 0\rangle$ and $\{10 \overline{1} 1\}\langle 11 \overline{2} 3\rangle$. Each CRSS of the secondary slip systems is from five to fifteen times as high as that of $\{01 \overline{1} 0\}\langle 11 \overline{2} 0\rangle$ in the low-temperature regime (Fig. 2). Subsequently, it was assumed that the CRSS of the secondary slip systems was ten times that of the primary or more. What the active of two secondary slip systems was distinguished, the ratio of either system was chosen as fifteen. In Types 5 and 6 , the ratio of the CRSSs in the three systems was given by the estimated values at 300 and $77 \mathrm{~K}$, respectively. The CRSSs of $\{10 \overline{1} 1\}\langle 11 \overline{2} 3\rangle$ and $\{01 \overline{1} 0\}\langle 11 \overline{2} 0\rangle$ were derived from the experimental data for single crystal $\alpha$-titanium, ${ }^{12,15)}$ although it is hard to evaluate the CRSSs of $\{0001\}\langle 11 \overline{2} 0\rangle$ and $\{10 \overline{1} 1\}\langle 11 \overline{2} 3\rangle$ at low temperatures due to deformation twinning. ${ }^{14)}$ Then the CRSS of $\{0001\}\langle 11 \overline{2} 0\rangle$ below $200 \mathrm{~K}$ was estimated by the $\tau_{\text {basal }}\left(=\tau^{*}+\tau^{\prime}\right)$, where the thermal $\tau^{*}$

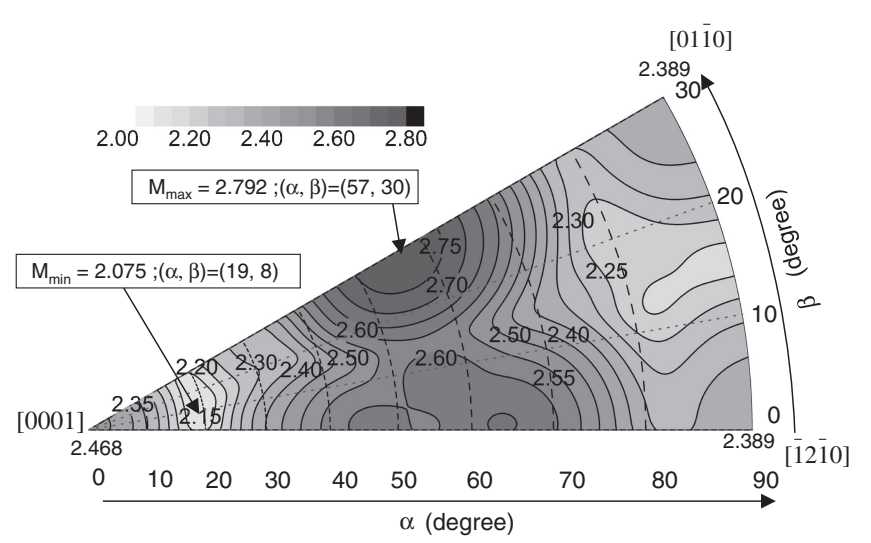

Fig. 3 Dependence of Taylor factor on tensile axis under the condition of homogeneous primary slips.

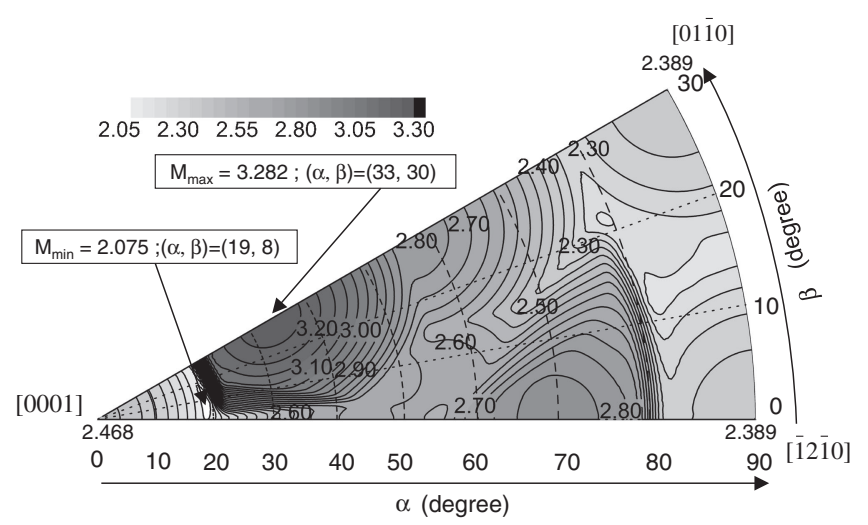

Fig. 4 Dependence of Taylor factor on tensile axis under the conditions of Types 2, 3, and 4.

and athermal component $\tau^{\prime}$ were given by the thermal activated process. The CRSS of $\{10 \overline{1} 1\}\langle 11 \overline{2} 3\rangle$ was also given by the data of Ti- $6 \mathrm{~mol} \% \mathrm{Al} .^{12)}$

\section{Results}

\subsection{Influence of slip systems on Taylor factor}

When the CRSSs of the active slip systems are equivalent (Type 1), $M_{\max }$ is 2.792 at the tensile axis with $(\alpha, \beta)=$ $(57,30)$ and $M_{\min }$ is 2.075 with $(\alpha, \beta)=(19,8)$ as shown in Fig. 3. The $M$ value is $2.473 \sim 2.792$ in the region of $\alpha=45 \sim 60^{\circ}$ and there is no big difference between $M_{\max }$ and $M_{\min }$. Thus, the factor $M$ is almost isotropic in any crystallographic orientation in Type 1 .

When the primary slip system is given as $\{01 \overline{1} 0\}\langle 11 \overline{2} 0\rangle$ and regardless of Types 2-4, no difference of $M$ and its dependence on the tensile axis is present (Fig. 4). $M_{\max }$ is 3.282 at the tensile axis with $(\alpha, \beta)=(33,30)$ and $M_{\min }$ is 2.075 with $(\alpha, \beta)=(19,8)$. In the tensile axes with $\alpha<19^{\circ}$ and $\alpha>82^{\circ}$, their $M$ factors are the same as that in Type 1 . In Types $2-4$, however, the $M$ factor is remarkably higher than that in Type 1 around the tensile axes with $(\alpha, \beta)=$ $(19,8)$ and $(\alpha, \beta)=(70,0)$. A high gradient in $M$, therefore, is produced at or near $\alpha=19^{\circ}$ and $\alpha=82^{\circ}$.

In the simple analysis by the Sachs model where only the primary slip system operated, the $M$ factor was $6.5 .{ }^{17)}$ When 

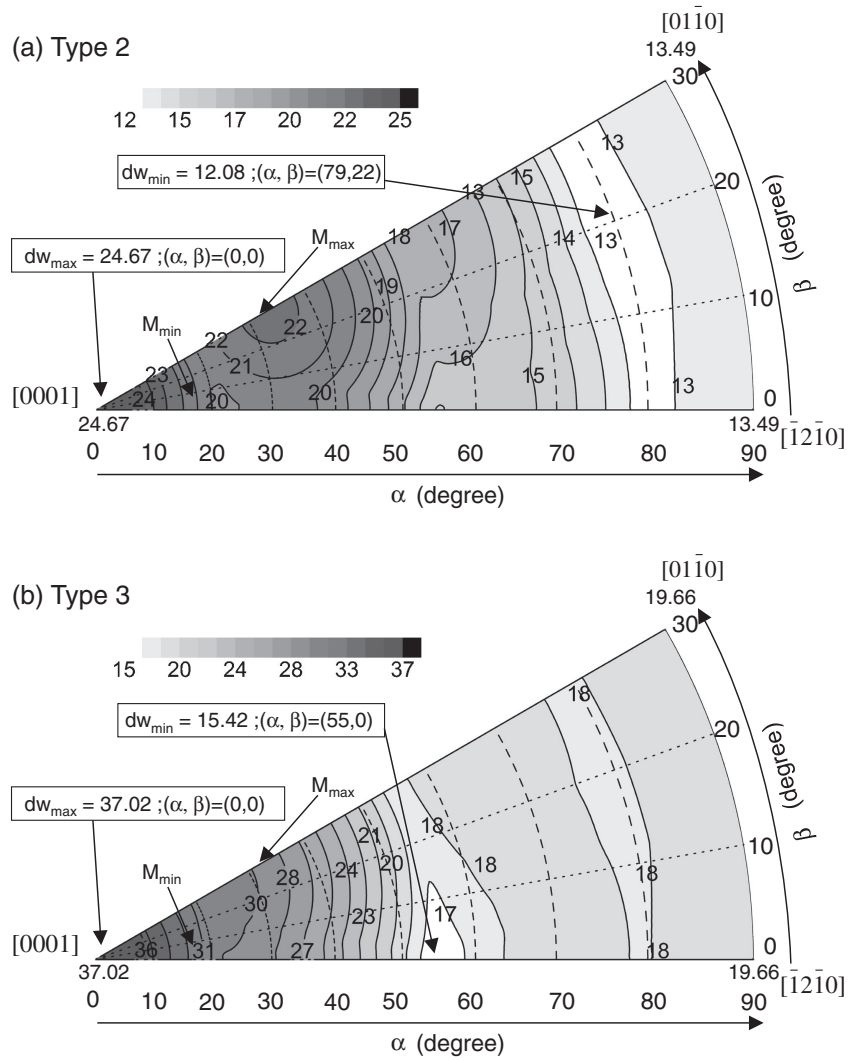

(c) Type 4

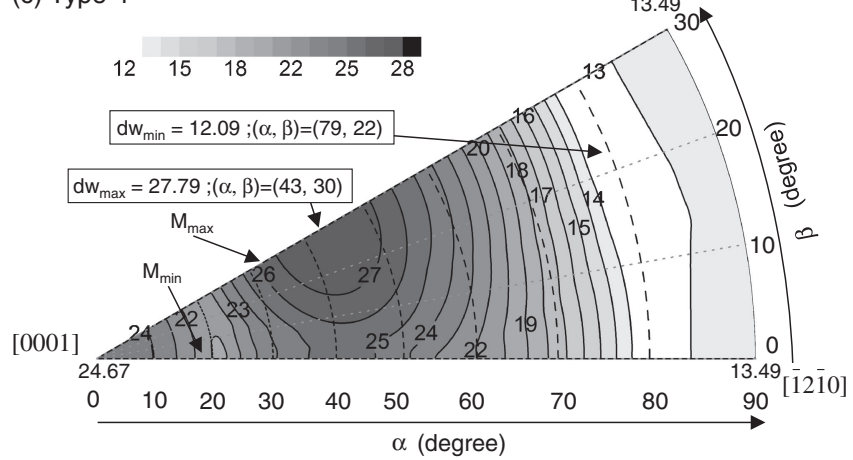

Fig. 5 Dependence of plastic work rate on tensile axis direction under the conditions of Types 2,3 , and 4 .

the homogeneous slip deformation is ideally available in a grain as in this study, the average $M, M_{\text {ave }}$, is about half the $M$ factor of the Sachs model on all tensile axes and types (Type 1: $M_{\text {ave }}=2.42$, Types $2 \sim 4: M_{\text {ave }}=2.63$, Types 5 6: $\left.M_{\text {ave }}=2.46\right)$.

\subsection{Influence of secondary slip systems on plastic work rate}

When $\{0 \overline{1} 10\}\langle 11 \overline{2} 0\rangle$ was chosen as the primary slip system, $\mathrm{d} w$ highly depended on the operating secondary slip systems. When $\{0001\}\langle 11 \overline{2} 0\rangle$ and $\{10 \overline{1} 1\}\langle 11 \overline{2} 3\rangle$ were operationally equivalent to secondary slip systems (Type 2), the tensile axes of the plastic work rate maximum, $\mathrm{d} w_{\max }$ and the minimum, $\mathrm{d} w_{\min }$ were different with those of $M_{\min }$ and $M_{\max }$, respectively (Fig. 5(a)): the $\mathrm{d} w_{\max }$ is 24.67 at $(\alpha, \beta)=(0,0)$, and the $\mathrm{d} w_{\min }$ is 12.08 at $(\alpha, \beta)=(79,22) . \mathrm{d} w$ continuously decreases as $\alpha$ increases from $0^{\circ}$ to $80^{\circ}$.

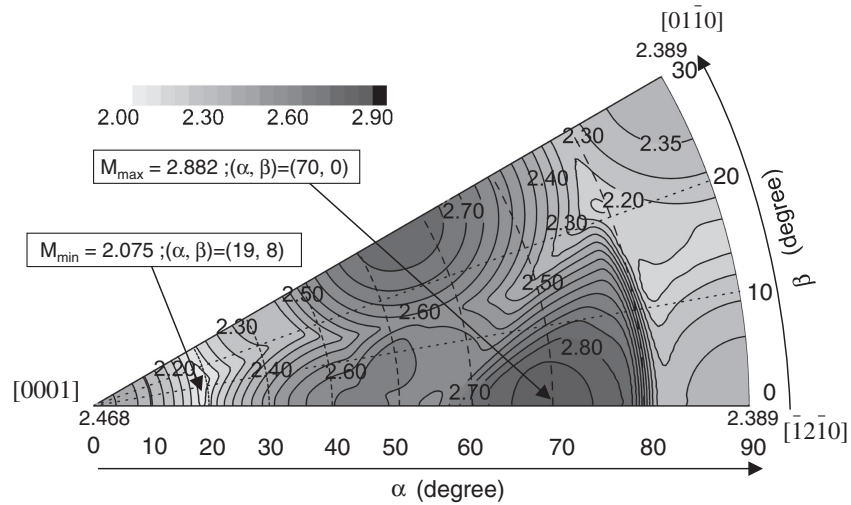

Fig. 6 Dependence of Taylor factor on tensile axis under the conditions of Types 5 and 6.

When $\{0001\}\langle 11 \overline{2} 0\rangle$ was chosen as the secondary slip system and the operation of $\{10 \overline{1} 1\}\langle 11 \overline{2} 3\rangle$ was prevented (Type 3), $\mathrm{d} w$ was higher than that of Type 2 in all of the tensile axes (Fig. 5(b)). d $w_{\max }$ is 37.02 at the same axis with $(\alpha, \beta)=(0,0)$ as in Type 2 , and $\mathrm{d} w_{\min }$ is 15.42 at $(\alpha, \beta)=$ $(55,0)$. The tensile axes of $\mathrm{d} w_{\max }$ and $\mathrm{d} w_{\min }$ are also not consistent with those of $M_{\min }$ and $M_{\max } . \mathrm{d} w_{\max }$ is 1.5 times as high as that in Type 2 and $\mathrm{d} w$ is 1.3 times at $\alpha=20 \sim 70^{\circ}$. At around $\alpha=55^{\circ}, \mathrm{d} w$ is almost the same with that in Type 2, therefore, the restriction of the $\{10 \overline{1} 1\}\langle 11 \overline{2} 3\rangle$ operation is not responsible for the plastic work rate.

When $\{10 \overline{1} 1\}\langle 11 \overline{2} 3\rangle$ was chosen as the secondary slip system and the operation of $\{0001\}\langle 11 \overline{2} 0\rangle$ was prevented (Type 4), $\mathrm{d} w$ increased in the region of $\alpha=20 \sim 70^{\circ}$ where the axis with $(\alpha, \beta)=(43,30)$ gave $\mathrm{d} w_{\max } 1.3$ times as high as that in Type 2 (Fig. 5(c)). Near the axes of $\alpha=0^{\circ}$ and $\alpha=90^{\circ}, \mathrm{d} w$ is almost the same with $\mathrm{d} w_{\min }$ and that in Type 2 .

\subsection{Slip deformation behavior of $\alpha$-titanium alloys at low temperature}

The dependence of the tensile axis direction on $M$ and $\mathrm{d} w$ was evaluated at $300 \mathrm{~K}$. The $300 \mathrm{~K} \mathrm{CRSS}$ of each slip system was adopted, as listed in Table 1 (Type 5). $M_{\max }$ is equal to 2.882 at $(\alpha, \beta)=(70,0)$, as shown in Fig. 6 , and $\mathrm{d} w_{\max }$ is equal to 54.92 at $(\alpha, \beta)=(0,0)$. $\mathrm{d} w$ continuously increases as the angle increases and equals 7.12 at $(\alpha, \beta)=(55,0)$. At $77 \mathrm{~K}$ (Type 6), $M$ is the same as that of Type 5 (Fig. 7(b)). Both $\mathrm{d} w_{\max }(74.03)$ and $\mathrm{d} w_{\min }(22.89)$ also have the same tensile axes as those in Type 5. Type 3 gave a similar ratio of CRSS with Types 5 and 6 . The results at $300 \mathrm{~K}$ and $77 \mathrm{~K}$ show almost the same dependence of $\mathrm{d} w$ on tensile axis with Type 3, although their tensile axis of $M_{\max }$ is different from that of Type 3.

\section{Discussion}

\subsection{Dependence of dominant slip system on tensile axis}

The $M$ factor around the tensile axes with $(\alpha, \beta)=(33,30)$ and $(\alpha, \beta)=(70,0)$ is higher in Types $2-4$, where $\{01 \overline{1} 0\}\langle 11 \overline{2} 0\rangle$ is the primary slip system (Fig. 4), than that in Type 1 where the CRSSs of three primary slip systems are 
(a) Type 5

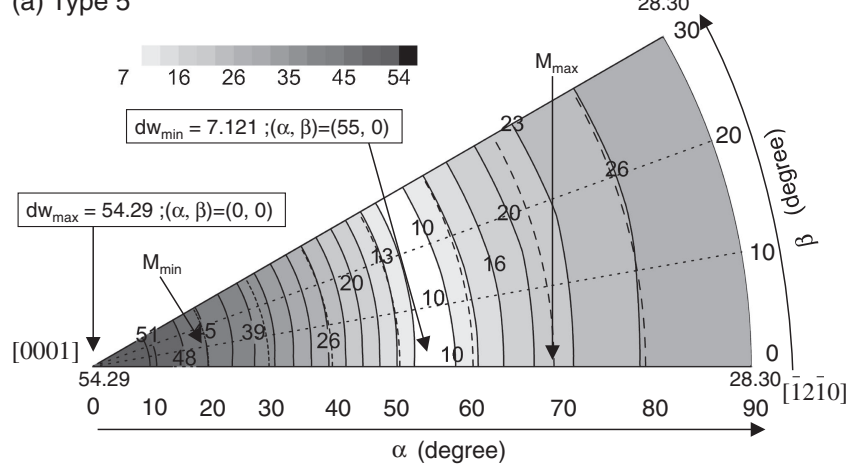

(b) Type 6

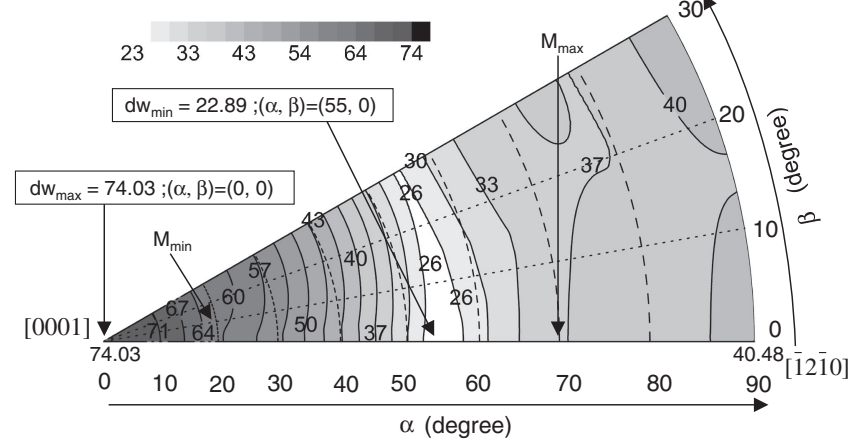

Fig. 7 Dependence of plastic work rate on tensile axis under the conditions of Types 5 (a) and 6 (b).

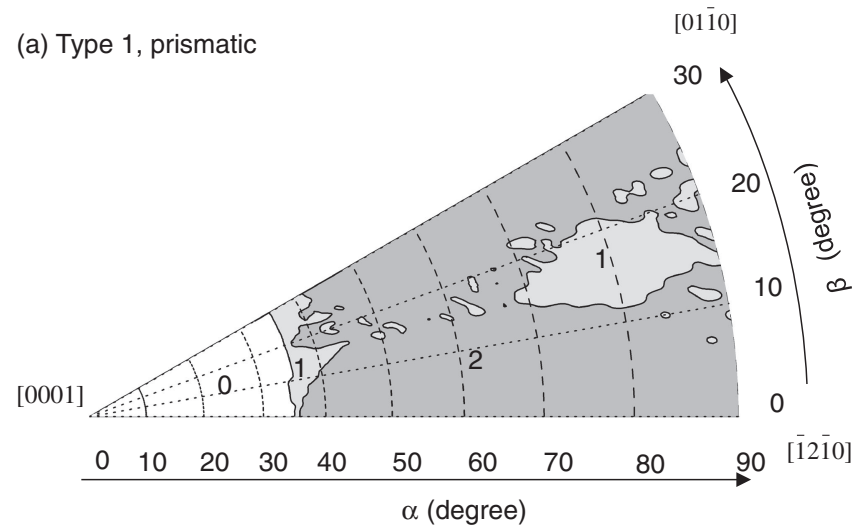

$[01 \overline{1} 0]$

(b) Types 2-4, prismatic

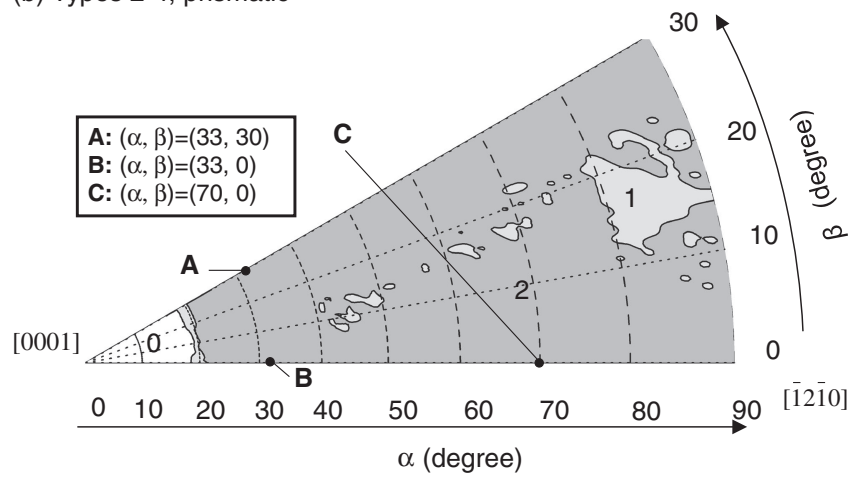

Fig. 8 Number of active prismatic slip systems under the conditions of Type 1 (a) and Types 2, 3, and 4 (b).

Table 2 Active five independent slip systems and their slip rates for tensile directions A, B, and C shown in Fig. 8(b).

\begin{tabular}{|c|c|c|c|c|c|c|}
\hline \multirow{4}{*}{ A } & A-1 & $(\overline{1} 100)[11 \overline{2} 0]$ & $(10 \overline{1} 0)[\overline{1} 2 \overline{1} 0]$ & $(0001)[11 \overline{2} 0]$ & (0001)[ī $\overline{1} \overline{1} 0]$ & $(01 \overline{1} 1)[2 \overline{1} \overline{1} 3]$ \\
\hline & $M=3.282$ & 0.898 & 0.257 & 0.204 & 0.553 & 1.370 \\
\hline & A-2 & $(\overline{1} 100)[11 \overline{2} 0]$ & $(10 \overline{1} 0)[\overline{1} 2 \overline{1} 0]$ & $(0001)[11 \overline{2} 0]$ & $(0001)[\overline{1} 2 \overline{1} 0]$ & $(01 \overline{1} 1)[\overline{1} \overline{1} 23]$ \\
\hline & $M=3.282$ & 0.257 & 0.898 & 0.553 & 0.204 & 1.370 \\
\hline & B & $(\overline{1} 100)[11 \overline{2} 0]$ & $(0 \overline{1} 10)[\overline{2} 110]$ & $(0001)[\overline{1} 2 \overline{1} 0]$ & $(\overline{1} 101)[2 \overline{1} \overline{1} 3]$ & $(01 \overline{1} 1)[\overline{1} \overline{1} 23]$ \\
\hline & $M=2.547$ & 0.257 & 0.257 & 0.664 & 0.685 & 0.685 \\
\hline & $\mathrm{C}$ & $(\overline{1} 100)[11 \overline{2} 0]$ & $(0 \overline{1} 10)[\overline{2} 110]$ & 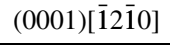 & $(1 \overline{1} 01)[2 \overline{1} \overline{1} \overline{1}]$ & $(0 \overline{1} 11)[\overline{1} \overline{1} 2 \overline{3}]$ \\
\hline & $M=2.882$ & 0.765 & 0.765 & 0.551 & 0.400 & 0.400 \\
\hline
\end{tabular}

the same (Fig. 3). It is suggested that the region of the tensile axis operating at $\{01 \overline{1} 0\}\langle 11 \overline{2} 0\rangle$ should be enlarged to increase its slip rates. In this section, the relationship between the operating slip system and slip rate is discussed.

The number of active slips of $\{01 \overline{1} 0\}\langle 11 \overline{2} 0\rangle$ in Types $2-4$ is increased from 0 to 2 at the tensile axis around $\alpha=33^{\circ}$ relative to Type 1 (Fig. 8). Because $\{01 \overline{1} 0\}\langle 11 \overline{2} 0\rangle$ has two independent slips, it seems to dominate the deformation around $\alpha=33^{\circ}$ in the tensile axis. However, the increase of $M$ depends on the $\beta$ angles as well (Fig. 4), and in that case the number of active slips in the primary system is not sufficient to discuss the dominant slip system during deformation. Here, the tensile axes, -indicated as points of $\mathrm{A}:(\alpha, \beta)=(33,30), \mathrm{B}:(\alpha, \beta)=(33,0)$, and $\mathrm{C}:(\alpha, \beta)=$ $(70,0)$ in Fig. 8 (b) were chosen to represent the axes that increased $M$ in Types $2-4$. All five active slip systems and their slip rates in Types $2-4$ were evaluated (Table 2).
Point A gives the axis of $M_{\max }$ in Types 2-4. $M_{\max }$ shows an increase of 0.941 relative to $M$ at the same axis in Type 1 . There are two sets of five independent slip systems for minimum work at the axis listed in Table 2 as A-1 and A-2. Each set involves two kinds of $\{01 \overline{1} 0\}\langle 11 \overline{2} 0\rangle$, i.e.,

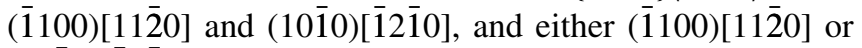
$(10 \overline{1} 0)[\overline{1} 2 \overline{1} 0]$, revealing a large slip rate. However, their Schmid factors are the same and as low as $S=0.128^{16,18)}$ so that the $\{01 \overline{1} 0\}\langle 11 \overline{2} 0\rangle$ is geometrically hard to operate. In Types $2-4$, therefore, the restrictions of other slip systems result in the operation of $\{01 \overline{1} 0\}\langle 11 \overline{2} 0\rangle$ because of their higher CRSSs. The operation of $\{01 \overline{1} 0\}\langle 11 \overline{2} 0\rangle$ in tension along axis A should be accompanied by deformation along the $c$-axis. As a result, the slip rate of $\{10 \overline{1} 1\}\langle 11 \overline{2} 3\rangle$ may be high.

At tensile axis $B$, the Schmid factors of the two slip systems, $(\overline{1} 100)[11 \overline{2} 0]$ and $(0 \overline{1} 10)[\overline{2} 110]$ are the same 

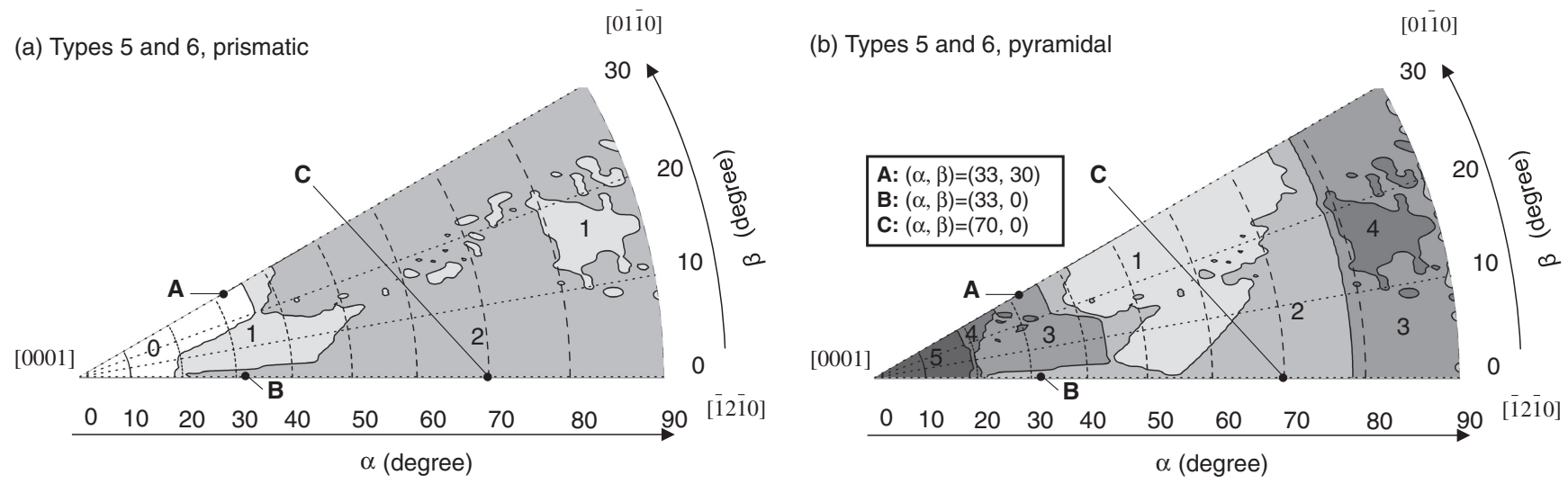

Fig. 9 Number of active slip systems under the conditions of Types 5 and 6 for prismatic (a) and pyramidal (b).

Table 3 Active five independent slip systems and their slip rates for tensile directions A, B, and C shown in Fig. 9.

\begin{tabular}{|c|c|c|c|c|c|c|}
\hline \multirow{4}{*}{ A } & A-1 & $(0001)[11 \overline{2} 0]$ & $(0001)[\overline{1} 2 \overline{1} 0]$ & $(10 \overline{1} 1)[\overline{2} 113]$ & $(\overline{1} 101)[2 \overline{1} \overline{1} 3]$ & $(01 \overline{1} 1)[2 \overline{1} \overline{1} 3]$ \\
\hline & $M=2.341$ & 0.416 & 0.556 & 0.685 & 0.549 & 0.136 \\
\hline & A-2 & $(0001)[11 \overline{2} 0]$ & $(0001)[\overline{1} 2 \overline{1} 0]$ & $(10 \overline{1} 1)[\overline{2} 113]$ & $(\overline{1} 101)[2 \overline{1} \overline{1} 3]$ & $(01 \overline{1} 1)[\overline{1} \overline{1} 23]$ \\
\hline & $M=2.341$ & 0.556 & 0.416 & 0.549 & 0.685 & 0.136 \\
\hline & B & $(\overline{1} 100)[11 \overline{2} 0]$ & $(0 \overline{1} 10)[\overline{2} 110]$ & $(0001)[\overline{1} 2 \overline{1} 0]$ & $(\overline{1} 101)[2 \overline{1} \overline{1} 3]$ & $(01 \overline{1} 1)[\overline{1} 1 \overline{1} 23]$ \\
\hline & $M=2.547$ & 0.257 & 0.257 & 0.664 & 0.685 & 0.685 \\
\hline & $\mathrm{C}$ & $(\overline{1} 100)[11 \overline{2} 0]$ & $(0 \overline{1} 10)[\overline{2} 110]$ & $(0001)[\overline{1} 2 \overline{1} 0]$ & $(1 \overline{1} 01)[2 \overline{1} \overline{1} \overline{3}]$ & $(0 \overline{1} 11)[\overline{1} \overline{1} 2 \overline{3}]$ \\
\hline & $M=2.882$ & 0.765 & 0.765 & 0.551 & 0.400 & 0.400 \\
\hline
\end{tabular}

$(S=0.128)$ and equal to the ones at axis A. Furthermore, their slip rates are small. Thus, $\{01 \overline{1} 0\}\langle 1 \overline{1} 20\rangle$ is less conducive to deformation in total even though it becomes active.

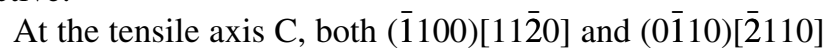
can easily operate, because their Schmid factors are high $(S=0.382)$ and $\{01 \overline{1} 0\}\langle 11 \overline{2} 0\rangle$ operates more easily when the secondary slip systems are suppressed.

In Types 5 and 6, the slip systems and their slip rates were also evaluated (Fig. 9 and Table 3). Because every active slip system and its slip rates in Types 5 and 6 are the same as those in Types $2-4$ at the tensile axes B and C, the deformation behavior of $\{01 \overline{1} 0\}\langle 11 \overline{2} 0\rangle$ in Types 5 and 6 is similar to those in Types 2-4. At the tensile axis A, however, $M$ is not higher than that in Type 1 . No $\{01 \overline{1} 0\}\langle 11 \overline{2} 0\rangle$ system is chosen, but three systems in $\{10 \overline{1} 1\}\langle 11 \overline{2} 3\rangle$ are active (Table 2). Therefore, $\{0001\}\langle 11 \overline{2} 0\rangle$ and $\{10 \overline{1} 1\}\langle 11 \overline{2} 3\rangle$ are dominant at the tensile axis $\mathrm{A}$.

Based on the above, the dominant deformation mode of $\{01 \overline{1} 0\}\langle 11 \overline{2} 0\rangle$ and the role of the secondary slip systems in the homogeneous deformation is discussed in sections 4.2 and 4.3 .

\subsection{Dominant deformation modes in primary slip sys- tems}

When all the CRSSs of the primary slip systems are the same (Type 1), $\{01 \overline{1} 0\}\langle 11 \overline{2} 0\rangle$ can hardly operate at the tensile axes around $\mathrm{A}$ and $\mathrm{C}$ where $M$ shows a steep incline. When the operation of $\{01 \overline{1} 0\}\langle 11 \overline{2} 0\rangle$ is predominant (Types
$2-4)$, both $\{0001\}\langle 11 \overline{2} 0\rangle$ and $\{10 \overline{1} 1\}\langle 11 \overline{2} 3\rangle$ are hardly active. Then, $\{01 \overline{10}\}\langle 11 \overline{2} 0\rangle$ must operate (Fig. 8(b)), and its slip rates has to increase. Therefore, $\{01 \overline{1} 0\}\langle 11 \overline{2} 0\rangle$ is the dominant mode. The same mode appeared in Types 5 and 6 , but the secondary systems of $\{0001\}\langle 11 \overline{2} 0\rangle$ and $\{10 \overline{1} 1\}\langle 11 \overline{2} 3\rangle$ are also dominant at the tensile axis A. At the tensile axis B in Types 2-6, the two slip systems of $\{01 \overline{10}\}\langle 11 \overline{2} 0\rangle$ are chosen (Figs. 8(b) and 9(a)) but also their slip rates are small (Tables 2 and 3 ). Therefore, it is not $\{01 \overline{10}\}\langle 11 \overline{2} 0\rangle$, but $\{0001\}\langle 11 \overline{2} 0\rangle$ and $\{10 \overline{1} 1\}\langle 11 \overline{2} 3\rangle$ that are the dominant modes at the $\mathrm{B}$ axis.

The tensile axis where the primary slip system $\{01 \overline{1} 0\}\langle 11 \overline{2} 0\rangle$ is dominant is evaluated. In Types $2-4$, the steep incline of $M$ at the tensile axes around $\alpha=18^{\circ}$ and $77^{\circ}$ (Fig. 4) must originate in the extension of the region where $\{01 \overline{1} 0\}\langle 11 \overline{2} 0\rangle$ is dominant.

The increase of $M$ from the condition of Type 1 is smaller at the tensile axes with $\alpha=40 \sim 77^{\circ}$, although the Schmid factor is higher. ${ }^{16,18)}$ In this regime, $M$ is comparatively high regardless of the secondary slip systems' operation, and the number of active $\{01 \overline{1} 0\}\langle 11 \overline{2} 0\rangle$ is mostly the same (Fig. 8). $\mathrm{d} w$ is also not so sensitive on the restrictions of the secondary slip systems (Fig. 5). At a tensile axis with $\alpha=40 \sim 77^{\circ}$, the secondary slip systems have less of an effect on the deformation, therefore, $\{01 \overline{1} 0\}\langle 11 \overline{2} 0\rangle$ is dominant.

Hence, the primary slip system $\{01 \overline{1} 0\}\langle 11 \overline{2} 0\rangle$ is the dominant mode at the tensile axes around $\mathrm{A}, \mathrm{C}$ and for $\alpha=40 \sim 77^{\circ}$, when the operation of the secondary slip systems is suppressed (Types 2 6). 
(a) Types 2-4, pyramidal

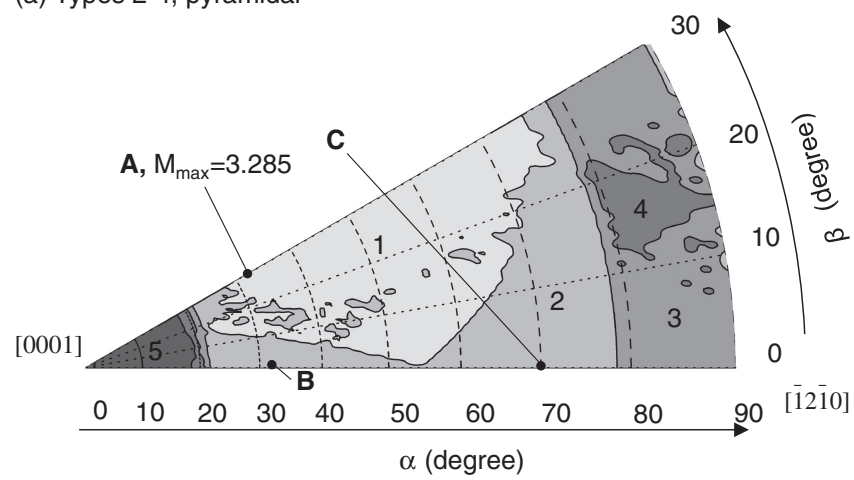

(b) Types 2-4, basal

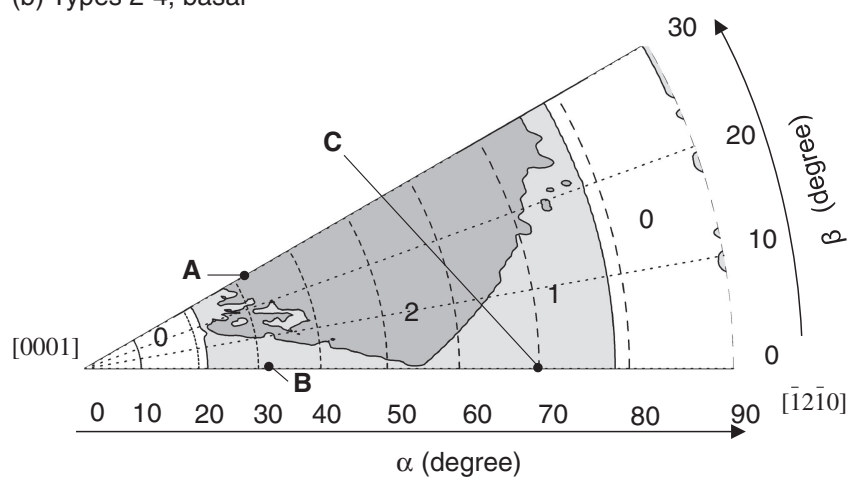

Fig. 10 Number of active slip systems under the conditions of Types 2, 3, and 4 for pyramidal (a) and basal (b).

\subsection{Role of secondary slip systems in homogeneous deformation}

The tensile deformation in which $\{01 \overline{10}\}\langle 11 \overline{2} 0\rangle$ is predominant is discussed. In Type 3 where the $\{10 \overline{1} 1\}\langle 11 \overline{2} 3\rangle$ operation is suppressed (Fig. 5(b)), $\mathrm{d} w$ is higher than that in Type 2 at all tensile axes (Fig. 5(a)). Because the operations of $\{0001\}\langle 11 \overline{2} 0\rangle$ and $\{10 \overline{1} 1\}\langle 11 \overline{2} 3\rangle$ are equivalent as the secondary slip system in Type 2 , the deformation in arbitrary shape needs an operating system of $\{10 \overline{1} 1\}\langle 11 \overline{2} 3\rangle$ at least (Fig. 10(a)). Furthermore, $\{10 \overline{1} 1\}\langle 11 \overline{2} 3\rangle$ is the dominant mode at the tensile axis at $\alpha=0 \sim 30^{\circ}$, because $\mathrm{d} w$ is remarkably higher there. Especially at the [0001] tensile axis, $M$ is relatively lower than the rest (Fig. 4), and the operating five slips are covered with only the $\{10 \overline{1} 1\}\langle 11 \overline{2} 3\rangle$ system at the tensile axis at $\alpha=0 \sim 20^{\circ}$ (Fig. 10(a)). Thus, an operation of $\{10 \overline{1} 1\}\langle 11 \overline{2} 3\rangle$ is necessary to deform homogeneously at the tensile axis around [0001]. However, it is difficult to observe $\{1 \overline{0} 11\}\langle 11 \overline{2} 3\rangle$ dislocations in tension along [0001] at low temperature. ${ }^{10)}$ Because the CRSS of $\{1 \overline{0} 11\}\langle 11 \overline{2} 3\rangle$ is increased at lower temperature, ${ }^{10,12)} \mathrm{d} w$ is higher at the tensile axis of [0001] (Fig. 7(b)).

In the tensile axes at $\alpha=77 \sim 90^{\circ}, \mathrm{d} w$ is not sensitive to the activity of $\{10 \overline{1} 1\}\langle 11 \overline{2} 3\rangle$ as shown in Fig. 5, although a few $\{10 \overline{1} 1\}\langle 11 \overline{2} 3\rangle$ systems are operative there (Fig. 10(a)). Namely, the $\{10 \overline{1} 1\}\langle 11 \overline{2} 3\rangle$ slip system is not dominant in the tensile axes at $\alpha=77 \sim 90^{\circ}$. In the tensile axes at $\alpha=$ $45 \sim 65^{\circ}$, other slip systems are dominant, because the activity of $\{10 \overline{1} 1\}\langle 11 \overline{2} 3\rangle$ is not responsible for $\mathrm{d} w$ (Fig. 5).

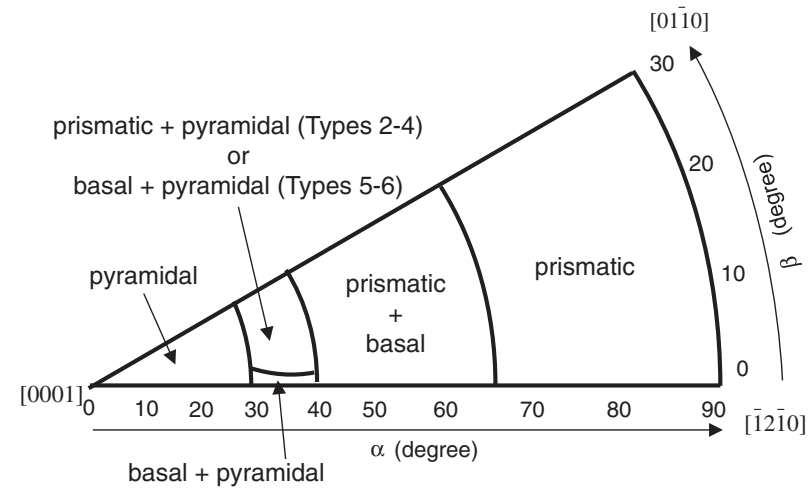

Fig. 11 Schematic illustration of dominant slip systems for tension in $\alpha$ titanium at low temperature.

The slip systems of $\{01 \overline{1} 0\}\langle 11 \overline{2} 0\rangle$ and $\{0001\}\langle 11 \overline{2} 0\rangle$ are mainly chosen in the tensile axes at $\alpha=45 \sim 60^{\circ}$ (Fig. 10). When the operation of $\{0001\}\langle 11 \overline{2} 0\rangle$ is suppressed (Type 4), $\mathrm{d} w$ in the tensile axes at $\alpha=30 \sim 65^{\circ}$ is higher than that in Type 2 where the secondary slip systems of $\{0001\}\langle 11 \overline{2} 0\rangle$ and $\{10 \overline{1} 1\}\langle 11 \overline{2} 3\rangle$ are operative (Fig. 5). $\mathrm{d} w_{\max }$ is also given at the tensile axis with $(\alpha, \beta)=(43,30)$ in Type 4 . Consequently, the $\{0001\}\langle 11 \overline{2} 0\rangle$ operation is dominant in the tensile axes at $\alpha=30 \sim 65^{\circ}$, but it is not active in all tensile axes (Fig. 10(b)). Thus, the $\{0001\}\langle 11 \overline{2} 0\rangle$ operation results in lowering $\mathrm{d} w$ and is not necessary for arbitrary deformation.

In tensile axes at $\alpha=77 \sim 90^{\circ}$, the secondary slip systems are not dominant. The primary slip system $\{01 \overline{1} 0\}\langle 11 \overline{2} 0\rangle$ that is dominant agrees with the high Schmid factor. ${ }^{17,18)}$

Figure 11 shows the dominant slip systems for arbitrary deformation, when $\{01 \overline{1} 0\}\langle 11 \overline{2} 0\rangle$ is the primary slip system. When the operation of the secondary slip systems is suppressed, plastic deformation hardly proceeded in the tensile axes at $\alpha=0 \sim 40^{\circ}$.

\subsection{Slip deformation in low-temperature high-cycle fatigue of $\alpha$-titanium alloy}

According to the analysis using the CRSSs of $\alpha$-titanium alloys at 77 and $300 \mathrm{~K}$ (Types 5 and 6), the operation of $\{01 \overline{10}\}\langle 11 \overline{2} 0\rangle$ and $\{0001\}\langle 11 \overline{2} 0\rangle$ is preferred in the tensile axes at $\alpha=40 \sim 65^{\circ}$, as shown in Fig. 10 where $\mathrm{d} w$ is low. However, the operation needs a large slip rate to produce unit strain in a grain, because their $M$ values are high. The slip rate may result from the increasing dislocation density so that the piled-up dislocations on $\{01 \overline{1} 0\}\langle 11 \overline{2} 0\rangle$ and $\{0001\}\langle 11 \overline{2} 0\rangle$ should readily occur. The analysis agrees with the experimental result in which piled-up dislocations of $\{01 \overline{1} 0\}\langle 11 \overline{2} 0\rangle$ were observed in the fatigued samples at $77 \mathrm{~K}^{2}{ }^{2}$

The operation of $\{10 \overline{1} 1\}\langle 11 \overline{2} 3\rangle$ is dominant at tensile axis with $(\alpha, \beta)=(0,0)$ where $\mathrm{d} w_{\max }$ is given. However, the operation of $\{10 \overline{1} 1\}\langle 11 \overline{2} 3\rangle$ is hard, because its CRSS is high at low temperature. In fact, the operation of $\{10 \overline{1} 1\}\langle 11 \overline{2} 3\rangle$ in the fatigued samples can be hardly observed by transmission electron microscopy. ${ }^{2)}$ Therefore, a large elastic strain field may accumulate under this condition. Facet or facets of $\{0001\}$ were detected in the subsurface fatigue crack initiation site of $\alpha$-titanium alloys. ${ }^{1-5)}$ It supports the development of opening stress (mode I) normal to $\{0001\}$. It is difficult to understand the transgranular cracking on 
\{0001\} due to the opening stress only. A few models of cracking mechanism have been proposed. One is transgranular cracking on $\{0001\}$ due to the combination of internal stress concentration and applied stress. ${ }^{19)}$ The other is the slip-off on $\{0001\}$ and its growth due to localized slip deformation. ${ }^{2}$ There is a strong possibility that slip-off occurs, because the development of dislocation pile-ups on $\{0001\}$ is preferred. However, no direct evidence of the cracking has been found.

In high-cycle fatigue fracture of $\alpha$-titanium alloys, the facet of $\{01 \overline{1} 0\}^{20)}$ or $\{11 \overline{2} 2\}^{21,22)}$ has been also detected at the crack initiation site. It was pointed out that there was a possibility to form the $\{01 \overline{1} 0\}$ facet due to the stress concentration with dislocation pile-ups of $\{01 \overline{1} 0\}\langle 11 \overline{2} 0\rangle$ and $\{0001\}\langle 11 \overline{2} 0\rangle$ as well as the $\{0001\}$ facet. Elastic stress can be accumulated normal to $\{10 \overline{1} 0\}$ at low temperature, because $\mathrm{d} w$ is comparatively high at the tensile axis at $\alpha=90^{\circ}$, as shown in Fig. 7. The cracking of $\{11 \overline{2} 2\}$ twin is different from that of $\{0001\}$ and $\{01 \overline{1} 0\}$ cracking, and may occur by another mechanism. An advanced deformation model that also considers twinning needs to be developed.

\section{Conclusions}

We evaluated the role of primary slip systems in tensile yielding and stress accumulation in $\alpha$-titanium alloys at low temperatures by using the Taylor model. The major conclusions are as follows:

(1) The primary slip system $\{01 \overline{1} 0\}\langle 11 \overline{2} 0\rangle$ was dominant in the tensile axes near $(\alpha, \beta)=(33,30)$ and $\alpha=40 \sim 90^{\circ}$.

(2) The operation of $\{0001\}\langle 11 \overline{2} 0\rangle$ decreased the plastic work rate in the tensile axes at $\alpha=30 \sim 65^{\circ}$ and was responsible for arbitrary deformation.

(3) The secondary slip system $\{10 \overline{1} 1\}\langle 11 \overline{2} 3\rangle$ was dominant in the tensile axes at $\alpha=0 \sim 30^{\circ}$. The operation of $\{10 \overline{1} 1\}\langle 11 \overline{2} 3\rangle$ was related to the deformation in all tensile axes and was necessary for arbitrary deformation.

(4) The plastic work rate was high at $(\alpha, \beta)=(0,0)$, because CRSS of $\{10 \overline{1} 1\}\langle 11 \overline{2} 3\rangle$ was high at low temperatures.
(5) The dislocation pile-ups of $\{0001\}\langle 11 \overline{2} 0\rangle$ and $\{01 \overline{1} 0\}\langle 11 \overline{2} 0\rangle$ may be the largest in the tensile axes at $\alpha=40 \sim 65^{\circ}$.

(6) The highest elastic stress may be developed in the tensile axis at $(\alpha, \beta)=(0,0)$ and may be responsible for (0001) transgranular cracking.

\section{REFERENCES}

1) O. Umezawa and K. Nagai: ISIJ Int. 37 (1997) 1170-1179.

2) H. Yokoyama, O. Umezawa, K. Nagai, T. Suzuki and K. Kokubo: Metall. Mater. Trans. A 31A (2000) 2793-2805.

3) M. R. Bache, W. J. Evans and H. M. Davies: J. Mater. Sci. 32 (1997) 3435-3442.

4) C. Sarrazin, R. Chiron, S. Lesterlin and J. Petit: Fatigue Fracture Eng. Mater. Struct. 17 (1994) 1383-1389.

5) V. Sinha, M. J. Mills and J. C. Williams: Metall. Mater. Trans. A 37A (2006) 2015-2026.

6) O. Umezawa, K. Nagai and K. Ishikawa: Tetsu-to-Hagane 76 (1990) 924-931.

7) M. Hamada and O. Umezawa: ISIJ Int. 49 (2009) 124-131.

8) W. F. Hosford: The Mechanics of Crystals and Textured Polycrystals, (Oxford Univ. Press, 1993) pp. 56-85.

9) G. Y. Chin and W. L. Mammel: Trans. Met. Soc. AIME 239 (1967) 1400-1405.

10) S. Hanada: Tetsu-to-Hagane 4 (1990) 495-502.

11) Notation for differentiation can express as both Leibniz's notation (d $w$, $\mathrm{d} \varepsilon$, and $\mathrm{d} \gamma)$ and Newton's notation $(\dot{W}, \dot{\varepsilon}$, and $\dot{\gamma})$. Leibniz's notation $(\mathrm{d} w, \mathrm{~d} \varepsilon$, and $\mathrm{d} \gamma)$ is used in this paper.

12) H. Numakura: Materia Japan 37 (1998) 117-124.

13) H. Conrad, M. Doner and B. Meester: Titanium Science and Technology, Vol. 2, (Plenum, New York, 1973) pp. 969-1005.

14) N. E. Paton and W. A. Backofen: Metall. Trans. 1 (1970) 2839-2847.

15) E. D. Levine: Trans. Met. Soc. AIME 236 (1966) 1558-1564.

16) F. Bridier, P. Villechaise and J. Mendez: Acta Mater. 53 (2005) 555567.

17) R. Armstrong, I. Codd, R. M. Douthwaite and N. J. Petch: Phil. Mag. 7 (1962) 45-58

18) F. Bridier, P. Villechaise and J. Mendez: Acta Meter. 56 (2008) 39513962.

19) M. R. Bache: Int. J. Fatigue 25 (2003) 1079-1087.

20) E. E. Sackett, L. Germain and M. R. Bache: Int. J. Fatigue 29 (2007) 2015-2021.

21) C. J. Beevers and M. D. Halliday: Metall. Sci. 3 (1969) 74-79.

22) Y. Ono, M. Demura, T. Yuri, T. Ogata, S. Matsuoka and S. Hori: Trans. Jpn. Soc. Mech. Eng. A 74 (2008) 329-334. 